however, some important differences in the way HMAX realizes the composition of features. For instance, the features used in HMAX are not encoded relative to one another in terms of their positions in space - they are combined, but without reference to where individual features fall in the image. This type of coding may seem problematic at first glance - one reaction is that HMAX could be 'fooled' into identifying a scrambled image with the appropriate features as an instance of a known object. Luckily, the set of features forming each view-tuned unit is 'overcomplete', that is, HMAX represents each object with an extremely large collection of different features that is highly redundant. Because each object is extremely over-specified, the odds of generating a scrambling in which all of the features describing an object are preserved is essentially zero. Moreover, this same coding redundancy allows HMAX to perform well at recognizing objects across configural deformations or across class instances. In both instances, although some of the features describing an object or class might be different, there are still many features that are likely to be consistent between what was learned and what is now being recognized.

Finally, HMAX offers an advance in how to pool over feature responses at each stage of processing. Earlier models that have proposed summing over differently weighted afferents to create progressively more complex detectors have typically relied on linear summation ${ }^{12}$. HMAX, in contrast, relies on the non-linear MAX operation. The MAX approach provides much of the robust generalization HMAX exhibits across changes in size and with images filled with visual clutter - for example, other objects in the scene. Importantly, MAX-like mechanisms are reflected in recent neurophysiological results - specifically, it has been observed that the response of a neuron to two features is related to its strongest activation to each of the features alone. Other results also support the idea that the responses of IT cells are often highly non-linear.

Interestingly, HMAX is in many ways a throwback to one of the earliest models of object recognition, a system referred to as "Pandemonium" that was proposed by Selfridge ${ }^{15}$ in 1959 . Pandemonium relied on a series of local feature detectors ('demons') that scanned each image for the presence of specific patterns, for example, a particular configuration of lines or an oriented curve. The responses of these feature detectors were then pooled to create more complex feature detectors at the next stage within the model. As with most theories that followed, summation across each layer of feature detectors was linear, with stronger responses at one layer being proportional to the number of appropriate features responding at the previous stage. Like Pandemonium, HMAX pools over progressively more complex detectors. However, unlike Pandemonium, these detectors are purely visual. Moreover, as illustrated in Figure 2, HMAX builds on what we now know regarding the response properties of successive layers in temporal cortex. Additionally, HMAX relies on the non-linear pooling of responses from one stage to the next. Even so, the general approach in Pandemonium and HMAX is markedly similar. Thus, everything old is new again. Yet because it so successfully addresses many of the perceived shortcomings of view-based models, HMAX truly is "News on Views."

1. Tarr, M. J. \& Bülthoff, H. H. J.Exp. Psychol.: Hum. Percept. Perform. 21, 1494-1505 (1995).

\section{Creating teetotaler mice}

The $\mathrm{GABA}_{A}$ receptor mediates the sedative and anxiety-reducing effects of ethanol. Although ethanol can potentiate $G A B A_{A}$ receptor function, the effects are variable, and it has been suggested that the receptor's sensitivity to ethanol may depend on its phosphorylation state. In this issue (pages 997-1002), Hodge and colleagues show that one isoform of protein kinase $C(P K C \varepsilon)$ mediates both the behavioral and biochemical response to ethanol by phosphorylation of the $\mathrm{GABA}_{\mathrm{A}}$ receptor. The authors found that mutant mice lacking PKC $\varepsilon$ show markedly reduced ethanol self-administration compared to wild-type mice, and are much more sensitive to the acute behavioral effects of ethanol. Biochemically, these effects are associated with an increased $\mathrm{GABA}_{\mathrm{A}}$ receptor sensitivity. Because the mutant mice consume less ethanol, the authors suggest that inhibitors of PKCE may be useful for treating alcoholism. Interestingly, the mutants also showed normal locomotor activity and did not appear to be sleepy or sedated. Inhibitors of PKC $\varepsilon$ could therefore also be a nonsedating alternative to enhance $G A B A_{A}$ function when treating disorders such as anxiety or epilepsy.

Kalyani Narasimhan
2. Biederman, I. \& Gerhardstein, P. C. J. Exp. Psychol. Hum. Percept. Perform. 21, 1506-1514 (1995).

3. Biederman, I. Psychol. Rev. 94, 115-147 (1987).

Logothetis, N. K. \& Pauls, J. Cereb. Cortex 3, 270-288 (1995).

Tarr, M. J., Williams, P., Hayward, W. G. \&

. Poggio, T. \& Edelman S. Nature 343,263-266 (1990).

7. Riesenhuber, M. \& Poggio, T. Nat. Neurosci. 2, 1019-1025 (1999).

8. Hubel, D. H. \& Wiesel, T. J. Physiol. 160, 106-154 (1962).

J. Neurophysiol. 66, 170-189 (1991).

10. Perrett, D. I., Rolls, E. T. \& Caan, W. Exp. Brain Res. 47, 329-342 (1982).

11. Ullman, S. Cognition 32, 193-254 (1989).

12. Perrett, D. I., Oram, M. W. \& Wachsmuth, E. Cognition 67, 111-145 (1998).

B Biol. Sci. 200, 269-294 (1978).

4. Bienenstock, E., Geman, S. \& Potter, D. in Advances in Neural Information Processing Systems 9 (eds. Mozer, M. C., Jordan, M. I. \& Petsche, T.) 838 (MIT Press, Cambridge, Massachusetts, 1997).

Selfridge, O. G. in Symposium on the Mechanisation of Thought Processes 513-526 (HM Stationery Office, London, 1959). 